\title{
Análisis de la triada: integración académica, permanencia y dispersión geográfica
}

\section{(Analysis of the triad: academic integration, permanence and geographic dispersion)}

\author{
Luis Moncada Mora \\ Fernando Negrete Zambrano \\ Max Arias Monteros \\ Ramiro Armijos Valdivieso \\ Universidad Técnica Particular de Loja (Ecuador)
}

DOI: http://dx.doi.org/10.5944/ried.22.1.22001

\section{Como referenciar este artículo:}

Moncada, L., Negrete, F., Arias, M. y Armijos, R. (2019). Análisis de la triada: integración académica, permanencia y dispersión geográfica RIED. Revista Iberoamericana de Educación a Distancia, 22(1), pp. 271-288. doi: http://dx.doi. org/10.5944/ried.22.1.22001

\section{Resumen}

Este artículo examina la relación que existe entre la decisión de permanecer o abandonar los estudios universitarios y el rendimiento académico inmediato, siguiendo el patrón de dispersión geográfica de la población estudiantil, que es una de las principales fortalezas de la educación a distancia. La información, base de la investigación, corresponde a una cohorte de estudiantes matriculados en una carrera de grado a distancia de la Universidad Técnica Particular de Loja (UTPL). Usando técnicas estadísticas y econométricas - principalmente modelos de probabilidad multinivel - se logró determinar que existe un efecto positivo del rendimiento académico inmediato en la decisión que toman los estudiantes de permanecer en los estudios. Este patrón de comportamiento se mantuvo en todos los centros de apoyo que tiene la UTPL, independientemente de su tamaño y distancia de la sede; es decir, la dispersión geográfica del centro de matrícula o lugar de vivienda del estudiante, no es un factor que influye en las decisiones que éste toma. La aversión a la pérdida es el principal factor de explicación del comportamiento, el hecho de no ser eficiente genera un efecto directo e inmediato, como es natural, no le encuentra sentido a su permanencia ya que no ha logrado integrarse adecuadamente.

Palabras clave: abandono estudiantil; integración; educación a distancia; modelo estadístico. 


\begin{abstract}
This article examines the relationship between the decision to stay or drop out of university and the immediate academic performance. This follows the pattern of geographic dispersion of student population, which is one of the main strengths of distance education. The information, the basis of the research, corresponds to a cohort of students enrolled in a distance learning degree program of the Universidad Técnica Particular de Loja (UTPL). Using statistical and econometric techniques - mainly multilevel probability models - it was possible to determine that there is a positive effect of the immediate academic performance in the decision that the students take to stay in the studies. This pattern of behavior was maintained in all the support centers of UTPL has, regardless of its size and distance from the headquarters; that is, the geographical dispersion of the student's enrollment center or place of residence is not a factor that influences the decisions it makes. The aversion to loss is the main factor explaining the behavior, the fact of not being efficient generates a direct and immediate effect, as it is natural, it does not find meaning in its permanence since it has not been properly integrated.
\end{abstract}

Keywords: Dropout; integration; distance education; statistical model.

La relación entre la permanencia y la integración académica tiene múltiples aristas de estudios, en el caso del sistema de estudios a distancia, el factor geográfico se suma como una variante de análisis e hipotéticamente hay la expectativa que no tenga una afectación en el comportamiento de los estudiantes. Se espera que, entre las tres variables, permanencia, integración académica y sector geográfico, exista una relación que permita entender el comportamiento de la permanencia y por tanto del abandono. El estudio de la permanencia resulta de singular interés porque afecta a una gran población estudiantil y también a las universidades que planifican idealmente sus trayectorias académicas, sin considerar aspectos reales que afectan fuertemente a las cohortes de estudiantes.

Tradicionalmente, al abandono estudiantil universitario se lo puede definir como la decisión personal que toma un estudiante de interrumpir su formación en el primer nivel, semestre o año de estudio (Bethencour, Cabrera, Hernández Cabrer, Álvarez y González, 20o8; Rode Larsen, Bjørnøy Sommersel y Søgaard Larsen, 2013; Moncada, 2014). Sin duda, el fenómeno del abandono temprano es crítico en el sistema de estudios, principalmente a distancia, porque genera altos costes reales y su efecto, a todo nivel, es negativo (Castaño, Gallón, Gómez y Vásquez, 2006; Willging y Johnson, 2009; Clerici, Giraldo y Meggiolaro, 2015; Juajibioy, 2016; Vallejos y Steel, 2016); por ello, disminuir la tasa de abandono temprano genera muchos retos y grandes esfuerzos.

"Ser estudiante en una universidad a distancia es bien distinto a serlo en la presencial” (Luque, García y De Santiago, 2014, p. 211). El factor diferenciador justamente es la distancia. Este argumento sirvió para definir que la investigación 
se enrumbaría en comprobar el efecto que, el rendimiento académico inmediato, utilizada como 'proxi' de la integración académica, tiene sobre el abandono temprano, así como examinar si existen diferencias en esta decisión en función al sector geográfico en el que se matriculó el estudiante. El sistema de estudios a distancia de la UTPL presentó las condiciones necesarias para hacer este análisis. La investigación se realizó con los datos de los estudiantes de la cohorte octubre 2016 - febrero 2017, de ellos se tomó la información de permanencia/abandono en el semestre abril - agosto 2017, rendimiento académico promedio en el primer semestre y lugar en el que habita, y se aplicó la técnica de análisis multinivel para modelos con variable dependiente cualitativa.

A priori, se espera que el rendimiento académico sea la variable que más aporta en el momento de explicar la decisión de permanencia. Asimismo, se cree que el sector geográfico en el que habita el estudiante no crea ningún tipo de diferencia, sino que sean las condiciones propias del estudiante las que generen los movimientos en la integración académica. En la presente investigación se comprobó que la "distancia" no es la causante del abandono de los estudios en la UTPL, sino que la aversión al fracaso, la poca eficiencia o el escaso nivel de integración académica influyen negativamente en la decisión de permanecer en los estudios universitarios, en este caso en los estudios a distancia de grado.

\section{¿LA DECISIÓN DE PERMANECER O ABANDONAR ESTÁ VINCULADA AL RENDIMIENTO ACADÉMICO INMEDIATO?}

Se entiende por rendimiento académico inmediato al resultado promedio de las calificaciones obtenidas por el almuno en un determinado periodo de tiempo, en este caso semestre (De Miguel \& Arias, 1999). En el caso del abandono temprano, la literatura especializada, por ejemplo, Himmel (2002), Araque (2009), Park y Choi (2009), Willging, Johnson (2009), Bernardo, Cerezo, Núñez, Tuero y Esteban (2015), Zotti (2015), Bernardo y otros (2016) y Juajibioy (2016) señalan que es un fenómeno multicausal, pero que la mayor cantidad de información, para explicar sus movimientos, la aporta el grado de integración académica que logra el estudiante en este primer espacio de tiempo de permanencia en la universidad. Por tanto, la relación causal se resume en el hecho de que un estudiante de nuevo ingreso tomará la decisión de permanecer en función de los resultados o notas obtenidas en su primera matrícula en la universidad. Ser eficiente, en este primer momento, resulta fundamental, ya que que es la fuerza impulsora que motiva a las personas a tomar una decisión de largo plazo, su esfuerzo y responsabilidad se ven reflejados en los resultados cuantitativos. Sin duda, históricamente, la eficiencia inmediata, ha definido la buena integración de los estudiantes universitarios, esto puede ser explicado por la caracterización de este hecho estilizado presentado por Moncada (2014). 
Los movimientos de las dos variables, rendimiento académico y permanencia, establecen patrones que permiten concluir que la probabilidad de permanecer o abandonar tiene una relación directa con los resultados inmediatos. A medida que se incrementa el rendimiento académico, la probabilidad de permanecer también se incrementa. Lo anterior tiene lógica ya que los estudiantes encuentran sentido y motivación en el hecho de permanecer en la universidad porque los resultados son satisfactorios (Tabbodi, Rahgozar y Makki Abadi, 2015); en el caso contrario, que no tengan un rendimiento cercano al mínimo exigible, no encuentran razón al hecho de permanecer. Todas las personas tenemos aversión a las pérdidas y un caso latente es el del abandono temprano, esto tiene su fundamento en lo señalado por Thaler (2017, p. 68), "las pérdidas duelen aproximadamente el doble de lo bien que gustan las ganancias". Aunque el comportamiento humano o las decisiones humanas no necesariamente siguen un patrón, en este caso, es evidente que la mayoría de estudiantes encuentran o fundamentan su decisión en los resultados inmediatos y en la aversión al riesgo que implica mantenerse en los estudios (Hallsten, 2017), si los resultados no le generan una mayor satisfacción personal comparados con otras actividades de la vida diaria, el estudiante decide abandonar (Zapata y Perneth, 2016).

Generar un movimiento positivo en la eficiencia académica, medida a través del número de asignaturas que aprueba en comparación con el número de asignaturas matriculadas, es el reto de los actores del sistema académico. Este movimiento tiene un efecto altamente positivo que trasciende lo cuantitativo, generando en el estudiante la confianza y el optimismo necesario para poder integrarse académica y socialmente al entorno universitario (Roso-Bas, Pades y García-Buades, 2016). Solo si el estudiante se siente satisfecho decidirá avanzar en la trayectoria académica que su carrera le plantea.

\section{LA DISPERSIÓN GEOGRÁFICA DE LA POBLACIÓN ESTUDIANTIL EN EL SISTEMA DE ESTUDIOS A DISTANCIA}

El sistema de estudios universitarios a distancia tiene como núcleo de acción al estudiante, al que el resto de elementos le proporcionan los recursos para facilitar la acción educativa (Rubio, 2014). La flexibilidad, entre otras significativas características, hace que esta modalidad tenga grandes poblaciones y esté conformada por un perfil estudiantil heterogéneo. Asimismo, el principio de igualdad de oportunidades se vive a través de la democratización del acceso a la educación de poblaciones tradicionalmente excluidas. Siguiendo la noción de distancia presentada por García Aretio (2001), a la "educación a distancia" se la vincula con la dispersión geográfica de la población estudiantil, las personas que viven en áreas alejadas de centros o instituciones educativas, universidades en este caso, ven como su mejor posibilidad este sistema de estudios; con este hecho el principio de igualdad de oportunidades no se ve lesionado en estos individuos. Incluso viviendo en las 
ciudades, miles de personas buscan un sistema de educación que se ajuste a sus necesidades laborales y familiares.

Llegar con la universidad a todas las poblaciones es parte fundamental de los objetivos de la formación a distancia (García Aretio, 2001), las barreras geográficas no existen en este sistema de estudios, y, por tanto, la expectativa es que el logro de los aprendizajes no esté restringido al área geográfica en la que habita el estudiante. Luque et al. (2014), en un estudio para la Universidad Nacional de Educación a Distancia de España (UNED), señalan que la 'distancia' no es el factor o mayor factor que causa el abandono, sino más bien el hecho de no poder armonizar las condiciones personales, familiares y laborales del estudiante. Independientemente de esta condición, el sector geográfico, se esperan resultados homogéneos de rendimiento académico, que este vector obedezca a sus propias condicionantes y no necesariamente al hecho de vivir en determinado lugar o sector geográfico. El anidamiento, resultado de la relación probabilidad de permanencia en función del rendimiento académico, no debería tener influencia por el sector geográfico el que habita el estudiante. En términos generales, no habría ningún tipo de agrupamiento o clúster.

\section{METODOLOGÍA}

La hipótesis propuesta es que el rendimiento académico inmediato explica el comportamiento de la permanencia/abandono temprano y que existen diferencias significativas en el comportamiento regional. Las carreras, agrupadas en cuatro áreas de estudio y soportadas por la gestión delos centros de apoyo dela Universidad Técnica Particular de Loja -UTPL- fue el entorno en el que se desarrolló la investigación y en el que se sometió a prueba la hipótesis. La información proviene de la cohorte de 6142 estudiantes de grado del sistema de estudios a distancia, matriculados en el periodo octubre 2016 - febrero 2017. Los 83 centros de apoyo -habilitados para ese periodo- cubren toda la geografía nacional, es decir, 24 provincias más los centros de apoyo internacionales en Madrid, Roma y Nueva York. De acuerdo al rumbo establecido en la investigación entraron en juego las siguientes variables de los estudiantes de grado: Centro de apoyo de matrícula, resultados de los aprendizajes medidos con el rendimiento académico promedio, y registros de matrícula.

La investigación utilizó un enfoque cuantitativo con una lógica deductiva. Además, es de tipo explicativo debido a que, a partir de modelos de comportamiento, se busca revelar las interacciones que se generan entre las variables propuestas. Para analizar la información cuantitativa se utilizaron herramientas de estadística descriptiva e inferencial. La exploración preliminar de la información permitió establecer que el análisis multinivel era la técnica adecuada para lograr los objetivos planteados. Este tipo de análisis ofrece la garantía de un examen exhaustivo de la información. Los datos multinivel se caracterizan por tener una estructura jerárquica, el ejemplo típico son los estudiantes anidados dentro de las aulas y 
aulas anidadas dentro de carreras o titulaciones. Los resultados de las pruebas de los estudiantes dentro de la misma clase pueden estar correlacionadas debido a la exposición al mismo maestro o libro de texto. La estructura que desvela el ejemplo, es el escenario de anidación de la información propia de este tipo de estudios (Cebolla, 2013; Winnaar, Frempong y Blignaut, 2015). En esta investigación, el anidamiento fue: los estudiantes matriculados en cada centro de apoyo y en cada zona de gestión en la que se distribuyen los centros de apoyo. En las formas de anidamiento establecidas se realizó un análisis descriptivo, principalmente gráfico, y una evidencia empírica con modelos de probabilidad simple y multinivel. Asimismo, los resultados del rendimiento académico se tomaron por cada asignatura en la que el estudiante se matriculó en el periodo académico de estudio. Cuantitativamente, el rendimiento académico se mide en una escala de cero a cuarenta puntos y en cada asignatura el estudiante debe alcanzar como mínimo 28 para aprobar la asignatura. Finalmente, manifestar que la información cuantitativa y cualitativa necesaria para la investigación se utilizó con el consentimiento del Vicerrectorado de Modalidad a Distancia de la UTPL. No se empleó información personal del estudiante, por lo que el sigilo está garantizado.

\section{RESULTADOS Y DISCUSIÓN}

Iniciamos describiendo el fenómeno de estudio. Para esta cohorte, la permanencia (47.88\%) fue inferior al abandono (52.12\%) (véase tabla 1) con una distribución homogénea en todas las carrera o titulaciones y en características personales -sexo y edad- de los estudiantes analizados. Vinculándolo con la teoría, el elevado indicador de abandono temprano, seguramente, tendrá su mayor explicación en los bajos resultados de rendimiento y no necesariamente en el sector en el que se matrícula y habita el estudiante.

Tabla 1. Proporción general de abandono y permanencia en la cohorte octubre 2016 febrero 2017

\begin{tabular}{|l|c|c|}
\hline \multicolumn{1}{|c|}{ Condición } & Frecuencia & Porcentaje \\
\hline Abandono & 3201 & 52.12 \\
\hline Permanencia & 2941 & 47.88 \\
\hline Total & 6142 & 100.00 \\
\hline
\end{tabular}

Un buen inicio para conocer la distribución de la tríada, abandono/permanencia, rendimiento académico y centro de apoyo, es conocer la dispersión inter e intracentro. En la figura 1 se evidencia la disposición de la información y la vinculación especial que existe entre estos tres elementos. 
Figura 1. Descripción de la varianza en el rendimiento académico inter e intracentro de apoyo. Diferenciada por grupos de permanencia y abandono

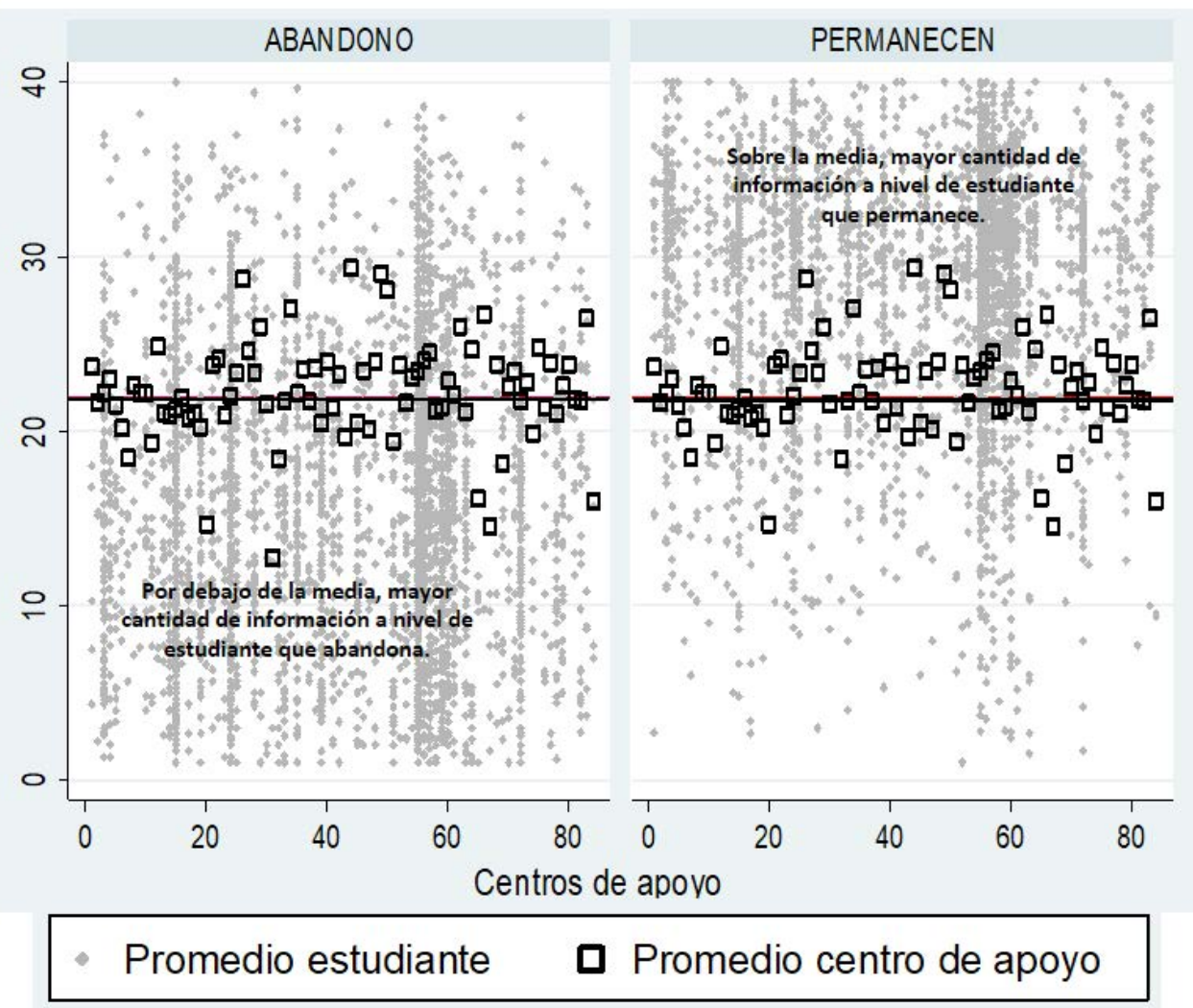

Para cada centro de apoyo -eje horizontal- se estudiaron: el promedio de rendimiento alcanzado por cada estudiante, el promedio de rendimiento de cada centro de apoyo y la media de toda la cohorte o media poblacional (21.92 de 40 puntos posibles). Con estas mismas características se diferenció a los estudiantes que abandonaron de los que permanecieron. El promedio de rendimiento de cada estudiante varía significativamente, pero, al diferenciar por la condición de abandono, la permanencia se agrupa por debajo del rendimiento poblacional en el caso del abandono y sobre el rendimiento poblacional en el caso de la permanencia. El rendimiento promedio de los estudiantes agrupados por centro de apoyo, se encuentra, en términos generales, bastante cercano al comportamiento poblacional. El escenario encontrado, es el primer indicador de que la variable dependiente probabilidad de permanencia- está determinada por el rendimiento de cada estudiante y no por el centro de apoyo. En el sistema de estudios a distancia, el lugar 
en el que se matricula el estudiante no debe influir en el rendimiento académico, asumiendo que el estudiante viene con competencias previas homogéneas.

En la búsqueda de posibles distorsiones, a la tríada anteriormente descrita, también incorporamos una diferenciación en el tamaño del centro de apoyo. El comportamiento puede estar influenciado por su tamaño (19 grandes, 64 pequeños).

Tabla 2. Resumen de medidas de tendencia y dispersión del rendimiento académico. Diferenciado por el tamaño de los centros de apoyo y la permanencia/abandono

\begin{tabular}{|l|c|c|c|c|c|c|c|c|}
\hline & \multicolumn{4}{|c|}{ Permanencia } & \multicolumn{4}{c|}{ Abandono } \\
\hline & Media & $\begin{array}{c}\text { Std. } \\
\text { Dev. }\end{array}$ & Min & Max & Media & $\begin{array}{c}\text { Std. } \\
\text { Dev. }\end{array}$ & Min & Max \\
\hline Grandes & 28.19 & .92 & 25.74 & 29.63 & 16.28 & 1.51 & 13.28 & 18.44 \\
\hline Pequeños & 28.10 & 3.25 & 18.21 & 34.75 & 16.70 & 4.22 & 8.67 & 34.40 \\
\hline
\end{tabular}

El rendimiento promedio es el mismo (28 puntos) para todos los centros cuando los estudiantes deciden permanecer. En el abandono, también, el promedio de los dos grupos - grandes y pequeños - es el mismo (16 puntos). La diferencia entre los dos grupos es la dispersión, los centros pequeños tienen una mayor separación de la media poblacional. La figura 2 confirma la dispersión moderada de los promedios de los estudiantes que están anidados en centros de apoyo pequeños, este comportamiento se da tanto en la permanencia como en el abandono. Esta figura también resulta de especial interés porque confirma la agrupación de la información en: rendimientos bajos característicos del abandono y rendimientos altos característicos de la permanencia. 
Figura 2. Descripción de la varianza en el rendimiento académico intracentro de apoyo. Diferenciada por grupos grandes y pequeños de centros de apoyo, y para los casos de los grupos que permanecen o abandonan

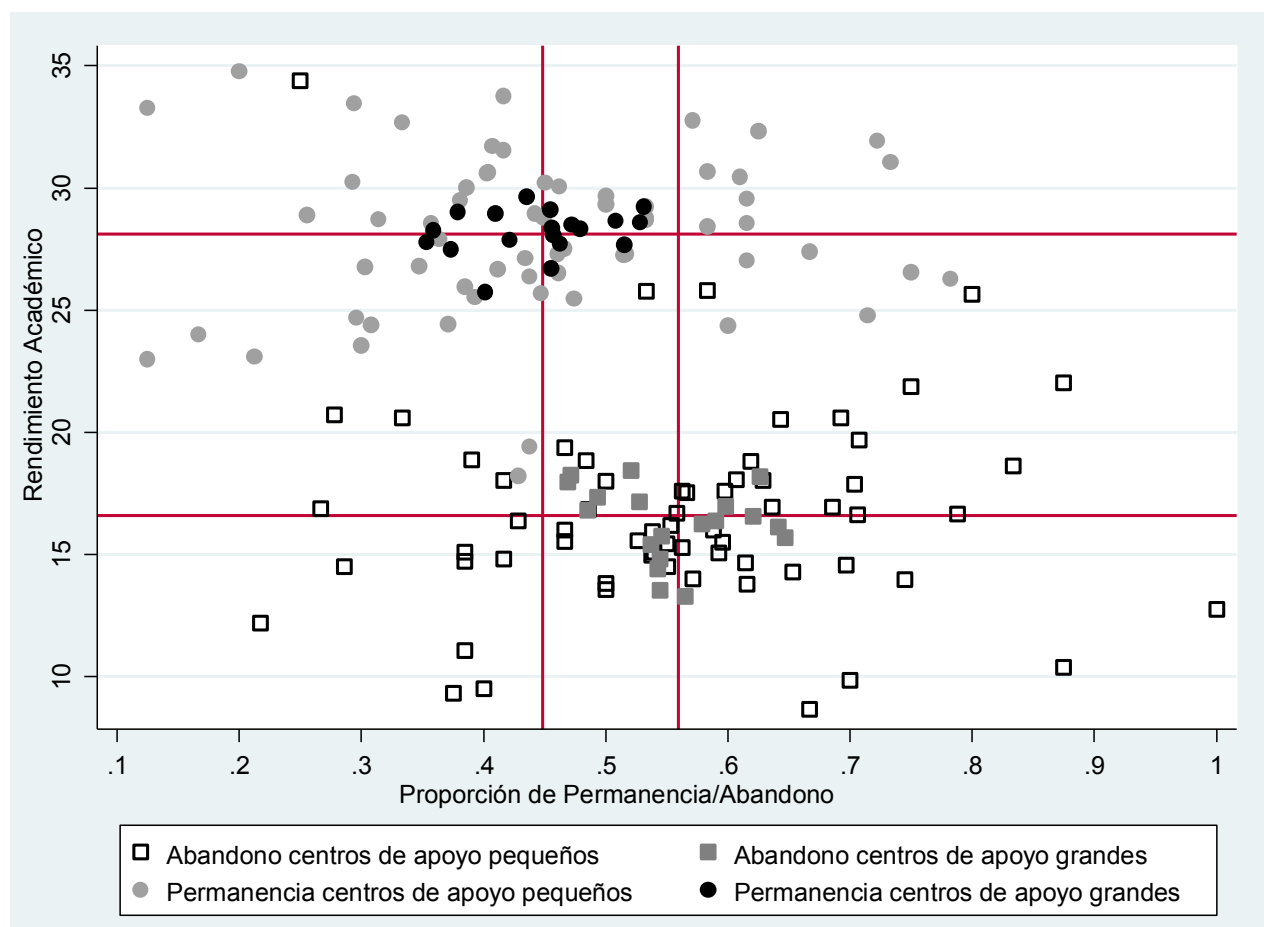

Para complementar el análisis y con la necesidad de encontrar el sentido de la dispersión intraclase presentada por los centros de apoyo pequeños, se agrupó la información de acuerdo a cinco regiones de gestión administrativa geográfica de la UTPL. La figura 3 recoge los movimientos que se presentan en estas regiones, los parámetros de comparación son los mismos que se emplearon para la descripción de la varianza -figura 2- en el rendimiento académico intracentro de apoyo. 
Figura 3. Varianza en el rendimiento académico intracentro de apoyo. Diferenciada por grupos grandes y pequeños de centros de apoyo, y para los casos de los grupos que permanecen o abandonan

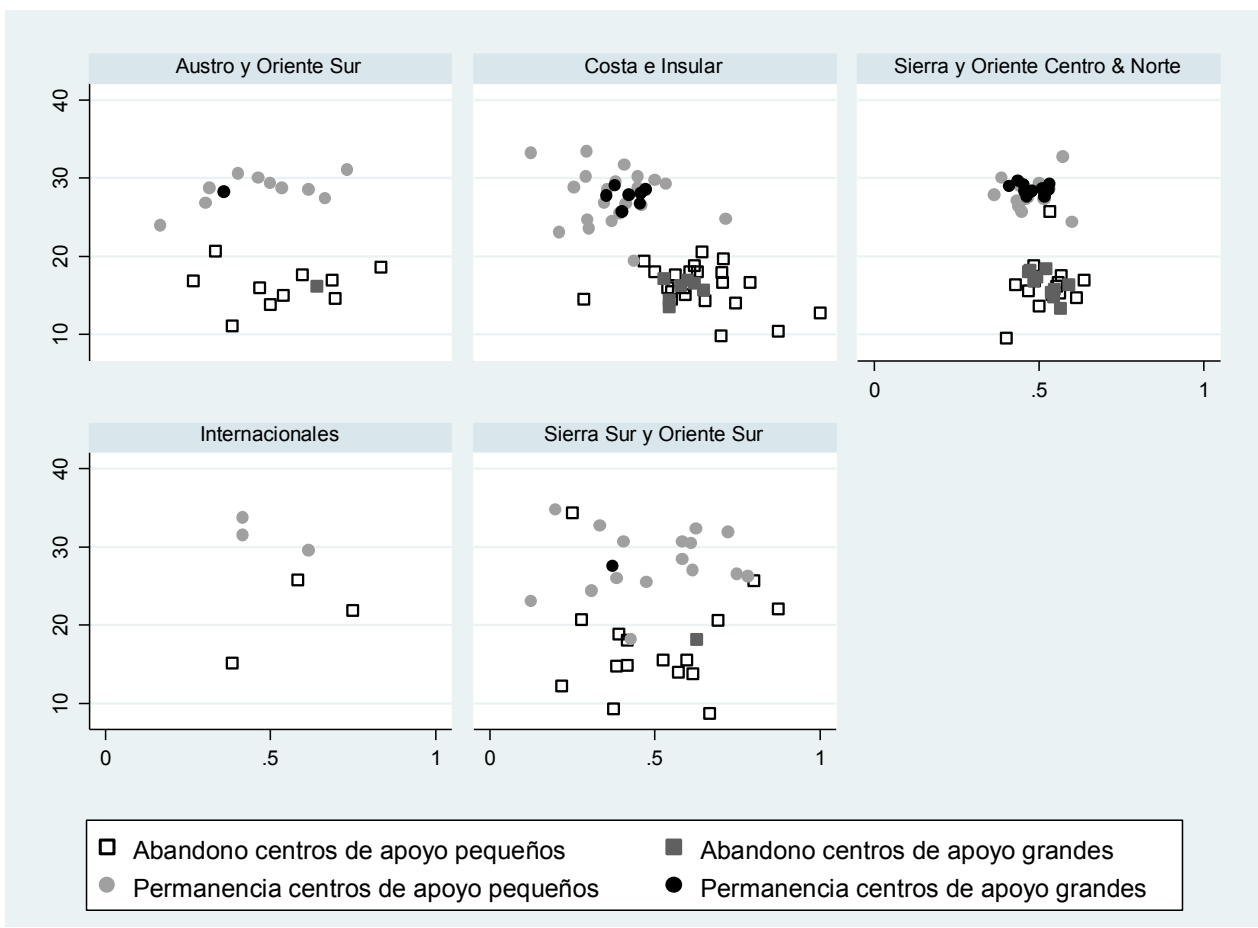

En efecto, las regiones de gestión se comportan como entes particulares. En las regiones densamente pobladas, el comportamiento de los grupos es homogéneo, en la figura 3 se muestra las regiones Sierra y Oriente Centro y Norte -principalmente-, y Costa e Insular. En las regiones con baja densidad de población estudiantil hay más dispersión del promedio intracentro de apoyo. Este hallazgo permitió reflexionar sobre un hecho que resulta coherente: en poblaciones pequeñas, los resultados promedio son muy sensibles a las variaciones.

$\mathrm{El}$ anidamiento de estudiantes por centro de apoyo sigue teniendo sentido, pero quizá se puede anticipar la conclusión de que la relación rendimiento académico y decisión de permanecer o abandonar es más una situación personal en la que no está involucrado el centro de apoyo. Al retomar la relación original, ampliamente discutida por la literatura especializada, la probabilidad de permanencia en función del rendimiento académico inmediato, encontramos que el comportamiento de los individuos agrupado en la figura 4, se ajusta a lo señalado en la caracterización teórica, los patrones de movimiento son positivos a la permanencia si el rendimiento 
académico sube y positivos al abandono -negativos a la permanencia- si el rendimiento académico baja. Los estudiantes que abandonan tienen un promedio de rendimiento bajo y los que permanecen un promedio que coincide con el mínimo aceptable por la UTPL. A priori, se cree que el rendimiento explique el comportamiento del abandono/permanencia, independientemente del centro de apoyo en el que está matriculado el estudiante.

Figura 4. Comportamiento del rendimiento académico promedio en función de la decisión de permanencia o abandono

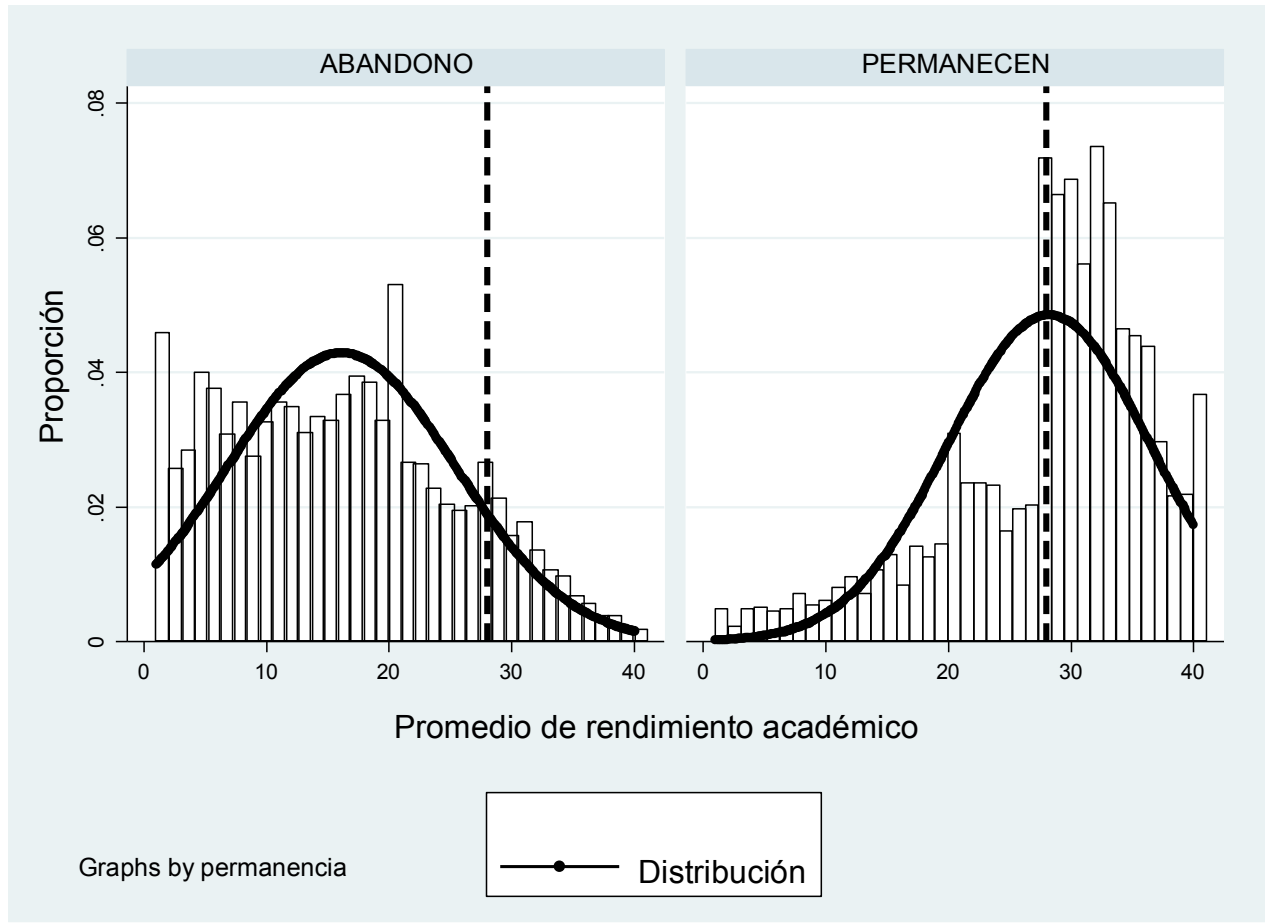

La relación propuesta para los tres elementos de análisis, matemáticamente, se puede resumir en las formas funcionales logit (Gujarati y Porter, 2010; Pindyck y Rubinfeld, 2001) y logit multinivel (Cebolla, 2013):

$$
\log \left[\frac{P_{i}}{1-P_{i}}\right]=\beta_{0}+\beta_{1} X_{i}+\beta_{1} D_{i}
$$$$
\log \left[\frac{P_{i j}}{1-P_{i j}}\right]=\gamma_{00}+\gamma_{1} X_{i j}+\mu_{0 j+\varepsilon_{i j}}
$$ 
En donde:

$P_{i} \quad$ Probabilidad de permanencia de cada estudiante

$X_{i} \quad$ Rendimiento académico promedio de cada estudiante

$D_{i} \quad$ Variable categórica de las regiones geográficas

$\gamma_{1} X_{i j} \quad$ Efectos fijos del modelo

$\mu_{o j} \quad$ Efecto aleatorio del grupo $\mathrm{j}$

$\varepsilon_{i j} \quad$ efecto aleatorio del individuo i del grupo $\mathrm{j}$

El supuesto de que el rendimiento académico inmediato explica los movimientos de la permanencia/abandono temprano y que existen diferencias en el comportamiento regional fue probado inicialmente con un modelo logit, y posteriormente con modelo logitmultinivel. Estas dos metodologías permitieron generar las relaciones necesarias entre las tres variables analizadas.

\section{RESULTADOS DEL MODELO DE PROBABILIDAD LOGÍSTICO}

Tabla 3. Resultados de las estimaciones. Modelo de probabilidad tipo logit. Efectos marginales del modelo logit. Modelo logit desagregando la variable región

\begin{tabular}{|l|c|c|c|c|}
\hline \multicolumn{1}{|c|}{ Permanencia } & Coeficiente & Std. Error. & $\mathrm{Z}$ & $\mathrm{P}>|\mathrm{z}|$ \\
\hline Promedio estudiante & $\mathbf{. 8}$ & .00 & 39.99 & .00 \\
PROMEDIO_EST(dy/dx) & $\mathbf{. 0 4}$ & $\mathbf{. o o}$ & $\mathbf{4 0 . 7 3}$ & $\mathbf{. 0 o}$ \\
Región & $\mathbf{. 0 7}$ & .04 & 2.13 & .03 \\
REGION(dy/dx) & $\mathbf{. 0 2}$ & $\mathbf{. o 1}$ & $\mathbf{2 . 1 3}$ & $\mathbf{. 0 3}$ \\
Intercepto & $\mathbf{- 4 . 3 0}$ & .14 & -30.60 & .00 \\
\hline Promedio estudiante & & & & \\
Región & $\mathbf{. 1 8}$ & .00 & 39.91 & .00 \\
COSTA E INSULAR & $\mathbf{. 0 3}$ & .11 & .31 & .76 \\
SIERRA Y ORIENTE C\&N & $\mathbf{. 2 4}$ & .11 & 2.31 & .02 \\
INTERNACIONALES & $\mathbf{- . 4 3}$ & .33 & -0.76 & .44 \\
AUSTRO YORIENTE SUR & $\mathbf{. 1 5}$ & .16 & .97 & .33 \\
Intercepto (SIERRA Y ORIENTE & $\mathbf{- 4 . 2 4}$ & .14 & -29.98 & .00 \\
S) & & & & \\
\hline Área entre la curva ROC & 0.86 & \multicolumn{5}{|l|}{ Correcta clasificación } & $78.77 \%$ & \\
\hline
\end{tabular}

Los resultados de la regresión logística binaria, resumida y presentada en la tabla 3 , sugiere, inicialmente, la existencia de efectos positivos de la variable predictora promedio. El rendimiento académico promedio del estudiante influye en .18 en el logaritmo de la razón de probabilidad de permanecer, con un efecto marginal del 4.40\%; la región, en cambio aporta a la explicación con un .07 y con un efecto marginal del $1.80 \%$. Para el análisis de la región se debe tener en cuenta la constante que en este caso es negativa $(-4.23)$ y hace que el efecto de la región en el logaritmo 
de la razón de probabilidad sea negativo. En este caso, la región es una variable politómica y teniendo en cuenta el análisis gráfico anterior, fue necesario estimar los efectos y la influencia o casuística que tiene cada región en la explicación de la razón de probabilidad de permanecer versus abandonar. Con excepción de la región Sierra y Oriente Centro y Norte, el resto de regiones tuvieron datos estadísticos poco significativos. Todas las regiones tienen diferentes propensiones y los efectos son positivos en tres de las cinco. Este hecho hace que la influencia geográfica en la decisión de permanecer o abandonar no se resuelva satisfactoriamente en esta primera evidencia.

\section{RESULTADOS DEL MODELO LOGÍSTICO MULTINIVEL}

Con los resultados del modelo de probabilidad logit, se concluyó que no existe un comportamiento tendencial marcado de las regiones y que la explicación, fundamentalmente, pasa por los resultados inmediatos. En este apartado se exponen y examinan los efectos de anidar la probabilidad permanencia de un estudiante a la región y al centro de apoyo en el que se matriculó. El objetivo de insistir en la revisión regional es descartar comportamientos extremos a todo nivel y confirmar algún comportamiento tendencial o inercial, más allá de la casuística, en determinado sector geográfico. En total, (véase tabla 4) se trabajó con 6142 observaciones, distribuidas en 5 regiones y 97 grupos creados con los 83 centros de apoyo. Los grupos creados son relativamente altos y heterogéneos en número, esto también fue evidente cuando gráficamente se distribuyó en centros de apoyo grandes y pequeños. La tabla 4, básicamente, agrupa los resultados de organización de las posteriores estimaciones. Los datos estadísticos de Wald chi2 y Log likelihood, en cualquiera de las dos formas de anidación, tienen similares resultados, lo que hace intuir que las varianzas son semejantes a cualquier nivel. Esto se confirmará con los resultados de las estimaciones.

Tabla 4. Resultados de la conformación de grupos para la estimación de los modelos logísticos multinivel.

\begin{tabular}{|l|c|c|c|c|}
\hline Observaciones & 6142 & & & \\
\hline Grupo variable & Grupos & Mínimo & Promedio & Máximo \\
\hline Región & 5 & 34 & 1228.40 & 3017 \\
\hline Centro apoyo & 97 & 1 & 63.30 & 705 \\
\hline & Wald chi2 & Prob > chi2 & Log likelihood & \\
\hline Región & 1578.96 & .00 & -2900.21 & \\
\hline Centro apoyo & 1595.82 & .00 & -2900.66 & \\
\hline
\end{tabular}


En el primer nivel de anidamiento, del modelo logístico multinivel, la región, a priori se esperan resultados similares en efectos y dispersión al modelo logit estimado anteriormente. El modelo tuvo los siguientes resultados:

Tabla 5. Resultados de la estimación del modelo logístico multinivel. Anidado a la región

\begin{tabular}{|l|c|c|c|}
\hline \multicolumn{1}{|c|}{ Permanencia } & Constante & Promedio estudiante & Región: Identity var(_cons) \\
\hline Coeficiente & $\mathbf{- 4 . 1 3}$ & $\mathbf{. 1 8}$ & .01 \\
\hline Std. Error & .12 & .00 & .01 \\
\hline$Z$ & -34.73 & 39.95 & [.0o .06] \\
\hline P $>|\mathrm{z}|$ & .00 & .00 & Prob $>=$ chibar2 = 0.06 \\
\hline \multicolumn{2}{l|}{ LR test vs. logistic model: } & chibar2(01) $=2.48$ & \\
\hline
\end{tabular}

Obsérvese en la tabla 5 que los efectos son similares a la regresión logística, en el caso del promedio es positivo (o.18) y la región representada por el intercepto es negativa (-4.13). Todos los estudiantes que tienen un rendimiento cero o nulo no tienen probabilidad de permanecer. Es decir, la razón de probabilidad de permanecer versus abandonar se mueve a partir de los cambios que pueda haber principalmente en el promedio. La dispersión o varianza (.oo) entre los grupos es muy baja, por lo tanto, la probabilidad de permanecer no se moverá significativamente por el hecho de estar matriculados en una determinada región, y no haber ningún patrón de agrupamiento. Esta primera forma de anidamiento es una evidencia sólida, pero, como se planteó en los resultados descriptivos y gráficos, es necesario llegar a un nivel de anidamiento menor para que se confirme la poca o nula influencia del sector geográfico en la decisión de los estudiantes.

El segundo nivel de anidamiento, del modelo logístico multinivel, es el centro de apoyo que pertenece a una determinada región. Los resultados se resumen en la tabla 6:

Tabla 6. Resultados de la estimación del modelo logístico multinivel. Anidado a la región y Centro de Apoyo

\begin{tabular}{|l|c|c|c|c|}
\hline \multicolumn{1}{|c|}{ Permanencia } & Constante & $\begin{array}{c}\text { Promedio } \\
\text { estudiante }\end{array}$ & $\begin{array}{c}\text { Región: } \\
\text { Identity var(_cons) }\end{array}$ & $\begin{array}{c}\text { Centro apoyo: } \\
\text { Identity var(_cons) }\end{array}$ \\
\hline Coeficiente & $\mathbf{- 4 . 1 2}$ & $\mathbf{. 1 8}$ & .00 & .02 \\
\hline Std. Error & .12 & .00 & .01 & .02 \\
\hline$Z$ & -34.89 & 39.74 & & [.00 .13] \\
\hline P $>|\mathrm{z}|$ & .00 & .00 & {$[.00 .10]$} & \\
\hline \multicolumn{2}{l}{ LR test vs. logistic model: } & chi2(2) $=3.39$ & Prob $>$ chi2 $=0.18$ & \\
\hline
\end{tabular}


Los resultados no son muy diferentes al primer nivel de anidamiento, de hecho, la constante y el promedio conservan los mismos efectos y valores similares. Se mantiene el hecho de que el mayor grado de explicación viene del lado del promedio de rendimiento y que la región no aporta explicación en ninguna de sus formas de anidamiento. En la región, el valor de la varianza es .oo y en el centro de apoyo .02, los cuales se pueden considerar como valores mínimos. Los intervalos de confianza son prácticamente cero; con lo que se confirma que no hay agrupamientos. El efecto que buscamos probar, es decir, la presencia de una perturbación aleatoria geográfica, no existe. La ubicación geográfica del estudiante no tiene ningún tipo de efecto en la decisión de permanecer o abandonar los estudios. Los resultados de la correlación intraclases, demuestran que tan solo un $.13 \%$ y .60\% de la varianza en la decisión de permanecer, es atribuible a las diferencias en las regiones y centros de apoyo. Las decisiones de los estudiantes de permanecer o abandonar no tienen un componente geográfico.

La poca estabilidad en el comportamiento regional hace evidente que la propensión a la permanencia es un tema cuya explicación, mayoritariamente proviene de los resultados y calificaciones inmediatas. La dispersión regional, o el lugar en el que habitan los estudiantes tiene poca o ninguna influencia en las decisiones que toman las personas que están en la faceta de estudiantes. Este primer resultado concuerda con lo expuesto en la fundamentación teórica, el componente principal de explicación de la decisión de los estudiantes es los resultados que le generan la confianza suficiente para continuar o avanzar en la trayectoria académica en la que se matricularon.

\section{CONCLUSIONES}

El análisis de la triada, permanencia/abandono, rendimiento académico y sector geográfico, ratifica la importancia de la integración académica como el factor que mayor información provee en el momento de explicar la decisión que toman los estudiantes. Las ecuaciones de comportamiento encontradas y expuestas en los resultados son la evidencia de que los estudiantes toman la decisión de permanecer a medida que sus resultados mejoran. Por ejemplo, en el primer modelo se establece que la probabilidad que un estudiante permanezca se incrementa en una tasa del 4.40\% a medida que el resultado promedio del estudiante mejora en un punto. Pese a ello, se debe tener en cuenta que este tipo de decisiones son movidas por razones o comportamientos humanos, como lo señala Thaler (2017), que relaciona el comportamiento con la aversión a las pérdidas. Un estudiante no es feliz cuando tiene resultados académicos pocos eficientes. Por lo tanto, parece natural que, en la disyuntiva de permanecer o abandonar los estudios universitarios, en este caso a distancia, la pérdida de las asignaturas, el bajo rendimiento, genere en el estudiante una reacción negativa y de aversión a la pérdida. No piensa en los beneficios en el largo plazo que le pueda traer el hecho de tener éxito en los estudios. 
El sistema de estudios a distancia necesita de una gran dispersión geográfica del lugar de matrícula. En este sentido, el mayor riesgo para la cohorte de estudiantes es que las competencias previas necesarias para que un estudiante tenga éxito no sean las mismas en todas las regiones, y esto afecte al rendimiento académico, consecuentemente se disminuya la probabilidad de permanencia de los estudiantes. El sector geográfico en el que habita el estudiante, tiene un efecto neutro en el rendimiento académico de este y, por tanto, en sus decisiones. La distancia no es el causante del abandono de los estudios en la UTPL. En la evidencia empírica desarrollada a través del análisis multinivel no se logró determinar comportamientos o agrupamientos de estudiantes que abandonan más en cierto sector geográfico.

Mejorar la permanencia y mitigar el abandono no es una labor sencilla. A partir de los resultados obtenidos se pudo concluir que la buena integración es el factor que genera el vínculo más fuerte del estudiante con su objetivo de profesionalizarse. Las universidades deben trabajar estrategias que faciliten la integración, todo esto debe ser en las primeras semanas de estudio y en el primer semestre. De la misma forma, las estructuras curriculares deben favorecer esta integración, de tal forma que el estudiante puede poco a poco insertarse en la vida universitaria.

\section{REFERENCIAS}

Araque, F., Roldan, C., y Salguero, A. (2009). Factors Influencing University Drop Out Rates. Computers \& Education, 53(3), 563-574.

Bernardo, A., Cerezo, R., Núñez, J. C., Tuero, E., y Esteban, M. (2015). Predicción del abandono universitario: variables explicativas y medidas de prevención. Fuentes, 16, 63-84.

Bernardo, A., Esteban, M., Fernández, E., Cervero, A., Tuero, E., y Solano, P. (2016). Comparison of Personal, Social and Academic Variables Related to University Drop-out and Persistence. Frontiers in Psychology, 1-9.

Bethencour, J. T., Cabrera, L., Hernández, J., Álvarez, P., y González, M. (2008). Variables psicológicas y educativas en el abandono universitario. Revista Electrónica de Investigación Psicoeducativa, 6(16), 603-622. Recuperado de http://www.alfaguia.org/ alfaguia/files/1320443945 45.pdf

Cabrera, L., Bethencourt, J. T., Alvarez, P., y González, M. (2006). El Problema del
Abandono de los Estudios Universitarios. The dropout problem in University Study. Relieve, 171-203.

Castaño, E., Gallón, S., Gómez, K., y Vásquez, J. (2006). Análisis de los factores asociados a la deserción y graduación estudiantil universitaria. Lecturas de Economía, 9-36.

Cebolla, H. (2013). Introducción al análisis multinivel. Madrid: CIS - Centro de Investigaciones Sociológicas.

Clerici, R., Giraldo, A., y Meggiolaro, S. (2015). The determinants of academic outcomes in a competing risks approach: evidence from Italy. Studies in Higher Education, 4O, 1535-1549.

De Miguel, M., y Arias, J. M. (1999). La Evaluación del Rendimiento Inmediato en la Enseñanza Universitaria. Revista de Educación, 320, 353-377.

García Aretio, L. (2001). La educación a distancia. De la teoría a la práctica. Barcelona: Ariel.

Gujarati, D. N., y Porter, D. C. (2010). Econometría. México D.F.: McGraw-Hill. 
Hallsten, M. (2017). Is Education a Risky Investment? The Scarring Effect of University Dropout in Sweden. European Sociological Review, 33(2),169-181.

Himmel, E. (2002). Modelos de análisis de la deserción estudiantil en la educación superior. Calidad de la Educación, 17, 91108.

Juajibioy, J. C. (2016). Study of University Dropout Reason Based on Survival Model. Open Journal of Statistics, 6, 908-916.

Luque, E., García, F., y De Santiago, C. (2014). El abandono y el egreso en la UNED. Instituto Universitario de Educación a Distancia, 211-218.

Moncada, L. (2014). La Integración Académica de los estudiantes universitarios como factor determinante del abandono de de corto plazo. Un ánalisis en el sistema de eduación superior a distancia del Ecuador. RIED. Revista Iberoamericana de Educación a Distancia, 17(2), 173-196.

Park, J.-H., y Choi, H. J. (2009). Factors Influencing Adult Learners' Decision to Drop Out or Persist in Online Learning. Educational Technology \& Society, 12(4), 207-217.

Pindyck, R. S., y Rubinfeld, D. L. (2001). Econometría: Modelos y Pronóstico. México, D.F.: Mc Graw Hill.

Rode Larsen, M., Bjørnøy Sommersel, H., y Søgaard Larsen, M. (2013). Evidence on Dropout Phenomena at Universities. Danish Clearinghouse for Educational Research. Recuperado de http://edu. au.dk/fileadmin/edu/Udgivelser/ Clearinghouse/Review/Evidence on dropout from universities brief version.pdf

Roso-Bas, F., Pades, A., y García-Buades, E. (2016). Emotional variables, dropout and academic performance in Spanish nursing students. Nurse Education Today, 37, 5358.

Rubio, M. J. (2014). Guía general de educación a distancia. Loja, Ecuador: EDILOJA.

Tabbodi, M., Rahgozar, H., y Makki Abadi, M. M. (2015). The Relationship between Happiness and Academic Achievements. European Online Journal of Natural and Social Sciences, 4(1), 241-246.

Thaler, R. (2017). Todo lo que he aprendido con la Psicología Económica. Bogotá: Deusto.

Vallejos, C. A., y Steel, M. F. (2016). Bayesian Survival Modelling of University Outcomes. Royal Statistical Society, 908916.

Willging, P. A., y Johnson, S. D. (2009). Factors that influence students' decision to dropout of online courses. Journal of Asynchronous Learning Networks, 13(3) 115-127.

Winnaar, L., Frempong, G. B., y Blignaut, R. (2015). Understanding school effects in South Africa using multilevel analysis: findings from TIMSS 2011. Electronic Journal of Research in Educational Psychology, 151-170. Recuperado de http://ojs.ual.es/ojs/index.php/EJREP/ article/view/1638

Zapata, D. O., y Perneth, A. (2016). Pedagogía y didáctica en la Fundación Universitaria Católica del Norte, elementos de la gestión del conocimiento que deben aportar a la permanencia estudiantil desde el humanismo y para la felicidad. Revista Reflexiones y Saberes, 3(5), 33-43.

Zotti, R. (2015). Should I stay or should I go? Dropping out from university: an empirical analysis of students' performances. AlmaLaurea, 1-26. Recuperado de https://www2.almalaurea.it/universita/ pubblicazioni/wp/pdf/wp70.pdf 


\section{PERFIL ACADÉMICO Y PROFESIONAL DE LOS AUTORES}

Luis Moncada Mora. Maestro en Economía Aplicada y Máster en Evaluación, Gestión y Dirección de la Calidad Educativa de la Universidad de Sevilla. Actualmente docente investigador de la Universidad Técnica Particular de Loja en la Titulación de Economía. Publicaciones relacionadas con Economía de la Educación.

E-mail:1fmoncada@utpl.edu.ec

Fernando Negrete Zambrano. Psicólogo Clínico, Pedagogo, Máster en política social y Máster en Sistemas Informáticos Educativos, con experiencia de trabajo en formulación, gestión y evaluación de proyectos sociales con énfasis en infancia, familia y desarrollo comunitario, docente universitario. Actualmente docente investigador de la Universidad Técnica Particular de Loja.

E-mail: jfnegrete@utpl.edu.ec

Max Arias Monteros. Doctor en Medicina Veterinaria y Zootecnica, Máster en Género Equidad y Desarrollo Sostenible. Actualmente docente investigador de la Universidad Técnica Particular de Loja en la Titulación de Economía.

E-mail: maarias2@utpl.edu.ec

Ramiro Armijos Valdivieso. Economista, Maestro en Economía Aplicada. Actualmente docente investigador de la Universidad Técnica Particular de Loja en la Titulación de Economía. Publicaciones relacionadas con Economía de la Educación. E-mail:prarmijos@utpl.edu.ec

Dirección:

Universidad Técnica Particular de Loja

Barrio San Cayetano Alto, calle Marcelino Champagnat s/n

Loja (Ecuador)

Fecha de recepción del artículo: 02/05/2018

Fecha de aceptación del artículo: 23/07/2018

Fecha de aprobación para maquetación: 11/11/2018 